\title{
Case study from the University of Kiel: breast feeding following breast cancer therapy
}

\author{
H. Kühling-von Kaisenberg, C. S. von Kaisenberg, K. Maass-Poppenhusen, W. Jonat \\ Department of Obstetrics and Gynecology, University of Schleswig-Holstein, Campus Kiel, Germany.
}

\section{Case report 1}

A 36-year-old premenopausal patient was initially diagnosed with breast cancer presenting as a lump in the upper quadrant of the right breast at 35 weeks of pregnancy. Subsequently she spontaneously delivered a healthy girl following induction of labor. Lactation was suppressed using Bromocriptin. Breast cancer was treated using neoadjuvant administration of four cycles epirubicin and cyclophosphamid (EC) as well as lumpectomy and axillary lymphonodectomy (invasive ductal breast cancer, pT2, pNo, M0, G2). Subsequently, four cycles of cyclophosphamid, methotrexate and 5 fluouracil (CMF) were given and the affected breast was irradiated (50 Gy).

Five years later, aged 41 , the lady spontaneously conceived. First trimester screening for chromosomal abnormalities and second trimester anomaly scanning showed no abnormal findings. At 32 weeks, the patient asked for counselling regarding the possibility to breastfeed. At 35 weeks, there was spontaneous rupture of the membranes and uneventful spontaneous delivery with normal APGAR and $\mathrm{pH}$ scores. Before the onset of lactation, a routine mammogram was performed, which was normal. The psychological circumstances of mammography, reminding the patient of her disease, resulted in stress and agalactia. With the support of midwives and lactation counsellers, normal lactation developed in the unaffected breast over a period of several weeks and the lady was breast feeding for 8 months. There was no lactation in the irradiated breast.

Correspondence to: Walter Jonat, Professor of Obstetrics and Gynecology, Head of Department, Department of Obstetrics and Gynecology, University of Schleswig-Holstein, Campus Kiel, Michaelisstrasse 16, 24105 Kiel, Germany. E-mail: jonat@email.uni-kiel.de

Received 20/06/05

Accepted 18/08/05

BCO $/ 413 / 2005 / \mathrm{CS}$

\section{Case report 2}

A 31-year-old patient was diagnosed with breast cancer after palpation of a nodule in the breast. The tumor was excised and axillary lymphonodectomy was performed (invasive ductal breast cancer, pT1, pN1 (2/11), M0, G2). Subsequently, 3 cycles of CMF were administered and irradiation therapy was performed (50 Gy).

Six years later, aged 37 , the patient received intracytoplasmic sperm injection (ICSI) for oligospermia of the husband due to malignancy of the testis, resulting in first trimester miscarriage. A further ICSI cycle lead to a normal pregnancy and delivery of a macrosomic healthy child through cesarean section. The patient also requested counselling regarding the possibility of breast feeding. Some days post partum, normal sufficient lactation developed in the healthy breast (Fig. 1), there was however no lactation in the irradiated side.

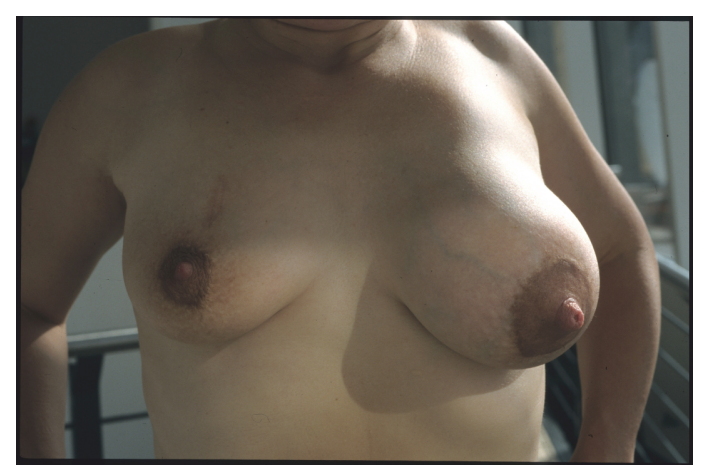

Figure 1.

Breast conserving surgical and irradiational therapy in breast cancer (right) and lactating breast (left). Surgical therapy was following functional and less cosmetic considerations to preserve the ducts, because at the time when the orientation of the skin cut was planned, there was no suspicion of malignancy. Therefore a radial rather than a circular cut was used. 
$Q$ - Do you think breast feeding can be generally recommended in breast preserving breast cancer therapy?

There is evidence, that breast feeding has favorable psychological effects in patients with breast cancer and can contribute to the establishment of a normal life. There is a protective effect of breast feeding before the onset of breast cancer. No data are available regarding a protective effect, once the diagnosis of breast cancer has been established.

$Q$ - How would you monitor the patient regarding recurrence of breast cancer during pregnancy and lactation?

Mammography may be used during pregnancy, but there may be technical limitations due to the density of the tissue. In general, $X$-rays should not be applied during pregnancy. Ultrasound is unharmful and can help detect lesions, or to distinguish solid from cystic lesions. In clinically suspicious cases magnetic resonance (MR) imaging may be used. The clinical diagnosis of mastitis at any stage of pregnancy should prompt investigations to exclude inflammatory breast cancer or recurrence. Postpartum, the window of 2-3 days before lactation may be used for mammography, therapy resistant mastitis or the detection of any suspicious lesion should in general result in weaning and should prompt immediate further investigations.

$Q$ - What is known about the long-term prognosis of breast cancer, if followed by pregnancy?

There are data suggesting that pregnancies following breast cancer improve the long-term outcome.
This may however be due to the 'healthy mother effect', suggesting that only the fittest after breast cancer also get pregnant.

$Q$ - Is it common, that irradiated breasts do not give milk subsequently?

Generally, as the result of irradiation, the lactating function of the breast has been stopped. In very few cases there may be reduced lactation.

\section{Further comment}

There is a very positive encouraging effect of breast feeding following breast cancer treatment, because it allows a return to a normal breast function and normal emotional relation with the breast as well as the positive psychological effect of pregnancy in general, such as the feeling to be able to give birth and produce life.

\section{Further reading}

1. Lawrence RA, Lawrence RM. Medical complications of the mother. In: Lawrence RA, Lawrence RM (Eds). Breastfeeding, 5th edition. St. Louis: Mosby; 1999; 525-527, 545-548.

2. Wilson-Clay B, Hoover K. Breast cancer. In: Wilson-Clay B, Hoover K (Eds). The Breastfeeding Atlas, 2nd edition. Texas, USA: LactNews Press; 2002; 96-101.

3. Higgins S, Haffty BG. Pregnancy and lactation after breast-conserving therapy for early stage breast cancer. Cancer 1994; 73: 2175-2180.

4. Kalache A, Vessey MP, McPherson K. Lactation and breast cancer. Br Med J 1980; 280: 223. 